\title{
Studying Close Approaches for a Cloud of Particles Considering Atmospheric Drag
}

\author{
Vivian Martins Gomes, ${ }^{1}$ Jorge Formiga, ${ }^{2}$ and Rodolpho Vilhena de Moraes $^{3}$ \\ ${ }^{1}$ Grupo de Dinâmica Orbital e Planetologia, Universidade Estadual Paulista-FEG/UNESP, Brazil \\ ${ }^{2}$ Instituto Nacional de Pesquisas Espaciais-INPE, Brazil \\ ${ }^{3}$ Universidade Federal de São Paulo/ICT, Brazil
}

Correspondence should be addressed to Vivian Martins Gomes; vivian.gomes@uol.com.br

Received 29 April 2013; Revised 22 July 2013; Accepted 26 July 2013

Academic Editor: Antonio F. Bertachini A. Prado

Copyright (C) 2013 Vivian Martins Gomes et al. This is an open access article distributed under the Creative Commons Attribution License, which permits unrestricted use, distribution, and reproduction in any medium, provided the original work is properly cited.

\begin{abstract}
The present paper has the goal of studying close approaches between a planet and a group of particles. The mathematical model includes the presence of the atmosphere of the planet. This cloud is assumed to be created by the passage of the spacecraft in the atmosphere of the planet, which can cause the explosion of the spacecraft. The system is assumed to be formed by the Sun, the planet, and the spacecraft that explodes and becomes a cloud of particles. The Sun and the planet are assumed to be in circular orbits and the motion is planar. The equations of motion are the ones given by the circular planar restricted three-body problem combined with the forces given by the atmospheric drag. In the numerical simulations, the planet Jupiter is the celestial body used for the close approaches. The initial positions and velocities of the spacecraft and the particles are specified at the periapsis, because it is assumed that this is the point where the explosion occurs.
\end{abstract}

\section{Introduction}

The close approach between a spacecraft and a celestial body received a lot of attention in the literature related to the astrodynamics field. Several missions used this idea to save fuel in their maneuvers. The Voyager mission visited several planets of the Solar System gaining energy from successive close approaches [1-4]. Other applications of this maneuver are available in the literature, like: the use of Swing-Bys in the inner Solar System to send a spacecraft to the giant planets [5] or even to the Sun [6]; the use of Venus in a trip to Mars $[7,8]$; studies to make a three-dimensional close approach to Jupiter to change the orbital plane of the spacecraft [9]; use of one [10] or two [11] passages by the Moon to increase the energy of the spacecraft; the use of multiple passages by the secondary body to find trajectories linking the primaries [12] or the Lagrangian points $[13,14]$.

A good description of this maneuver is available in Broucke [15]. There are many other researches available in the literature considering this problem, several of them optimizing parameters to obtain some desired results, like obtaining multiple close approaches [16-19], combining this maneuver with low thrust [20] or impulsive maneuvers [21]. The eccentricity of the primaries was also included in some researches, like in reference [22].

In the present paper, the effects of the atmosphere of the planet during the close approach $[23,24]$ between a planet and a cloud of particles are studied. The mathematical model is given by the circular restricted planar three-body problem [25], with the inclusion of the atmospheric drag. The cloud is assumed to be created by the explosion of the spacecraft during the closest approach, which is the point where it suffers the strongest effect from the atmosphere. Similar studies are considered in [26,27]. This same type of problem is considered from the astronomical perspective in references [28-33].

For each particle created by the explosion of the spacecraft, the equations of motion are numerically integrated forward in time, until a point where the particle is at a distance that can be considered far enough from the planet and it is possible to disregard the effects of the planet and consider the Sun-particle as a two-body system. A and B are 
the points where the particles and the spacecraft are assumed to be far from the planet. Then we can use equations from the two-body celestial mechanics to compute the velocity, energy and angular momentum after the passage by the planet, for each particle. From those results, the eccentricity and the semi-major axis of each particle can be obtained. After that, the orbit of the spacecraft can be integrated backwards in time, now as a single body. The planet is assumed to be in a circular orbit centered in the central body and any keplerian orbit is valid for the spacecraft. The difference from the standard close approach maneuver is the existence of an atmosphere in the planet and it causes a drag force in the spacecraft that makes the explosion and changes the trajectories of the particles after that explosion. The equations of motion are the ones of the restricted planar circular threebody problem [25] with the addition of the atmospheric drag, in the same way done for a single particle in reference [23]. The drag force is assumed to be proportional to the square of the velocity and the density of the atmosphere varies exponentially with the altitude. The main goal of the present research is to map the change of the orbits of the particles that form the cloud after the close approach with the planet. In particular, it is mapped the orbital parameters of the particles after passing by the planet. After that the effects are compared with similar maneuvers made without the presence of the atmosphere of the planet. This type of work is important, because it helps to obtain the size and density of the cloud of particles after the passage, for any given time. That information has a strong impact on the evaluations of the risks that spacecrafts suffer when passing by shorter distances from this cloud. It is also possible to use those informations to plan orbital maneuvers for a spacecraft to escape from the cloud or, at least, to pass by regions of smaller densities. The costs of those maneuvers are strongly dependent on the time in advance that it starts. So, by having a better knowledge of the size and density of the cloud ahead of time, it is possible to reduce the risk and the consumption of the maneuvers that will be realized to escape from the cloud.

\section{Definition of the Problem and Mathematical Model}

Since the main goal of the present paper is to study the evolution of a cloud of particles formed by the explosion of the spacecraft, the first hypothesis is that the spacecraft comes from outside the sphere of influence of the planet. Then, it enters in the atmosphere of that planet and it travels, as a single body, governed by the gravity and the atmosphere of the planet until it reaches the periapsis of its trajectory around the planet. Although this motion is not keplerian, there is always a point of closest approach to the planet that is called periapsis. At this point the effects of the atmosphere reach its maximum point, transferring energy to the spacecraft and increasing its temperature. It is then assumed that the protecting shield of the spacecraft fails and that it explodes, forming a cloud of particles. Those particles then follow independent trajectories, governed by the same forces (gravity and atmosphere of the planet). The planet is assumed to be travelling in a circular orbit centered in the Sun and the spacecraft comes to the close approach from an elliptic orbit, also centered in the Sun. Figure 1 illustrates this situation. The spacecraft is initially travelling in the elliptic orbit around the Sun that has a perihelion marked by the point P. Jupiter is travelling in a circular orbit around the Sun and it has a sphere of influence represented by the circle around its center. The point $\mathrm{A}$ is the position where the spacecraft starts its approximation to Jupiter, but it is still far from the planet such that the system can be modeled as a two-body problem (spacecraft-Sun). The spacecraft follows its trajectory, now under the gravitational forces of the Sun and Jupiter, until it enters the atmosphere of the planet, when the extra force generated by the drag is included in the dynamics. This force not only modifies the trajectory of the spacecraft, but it also generates the explosion of the spacecraft when it is passing by the point of the closest approach to the planet. After that, the cloud of particles follows its way in the atmosphere, as individual bodies. If there is no capture by the planet Jupiter, the particles will leave the planet and go back to an orbit around the Sun. Point B represents the exit point from the sphere of influence of Jupiter. It is a generic point, marked to explain the main ideas of the trajectory. Each particle has its own exact point of escaping, since the trajectories are independent from each other after the explosion. The equations of motion for each particle and for the spacecraft are the ones given by the planar restricted circular three-body problem with the inclusion of the atmospheric drag. Those equations are written in the rotating system of reference that is a system that rotates together with the primaries of the system (Sun and Jupiter in the present case). The canonical system of units is used. It implies that the unit of distance is the Sun-Jupiter distance, the unit of mass is the total mass of the bodies involved (Sun and Jupiter, since the spacecraft has a negligible mass) and the unit of time is defined in such a way that the period of the motion of the primaries is $2 \pi$. Under those hypothesis, the equations of motion are [23]:

$$
\begin{aligned}
& \ddot{X}-2 \dot{Y}=\frac{\partial \Omega}{\partial X}+F_{X}, \\
& \ddot{Y}+2 \dot{X}=\frac{\partial \Omega}{\partial Y}+F_{Y},
\end{aligned}
$$

where $\Omega$ is the potential, given by:

$$
\Omega=\frac{1}{2}\left(x^{2}+y^{2}\right)+\frac{(1-\mu)}{r_{1}}+\frac{\mu}{r_{2}}
$$

and $F_{x}$ and $F_{y}$ are the components of the drag force $(\vec{F})$, expressed by:

$$
\vec{F}=\left(F_{X}, F_{Y}\right)=\frac{-C_{D} A V \rho_{0} e^{-\left(h-h_{0}\right) / H}}{2 m} \vec{V},
$$

where $C_{D}$ is the drag coefficient, which is a constant that takes into account the form of the spacecraft or the particles; $A$ is the cross section area of the spacecraft or the particles; $\vec{V}$ is the velocity of the spacecraft or the particles; $m$ is its mass; 


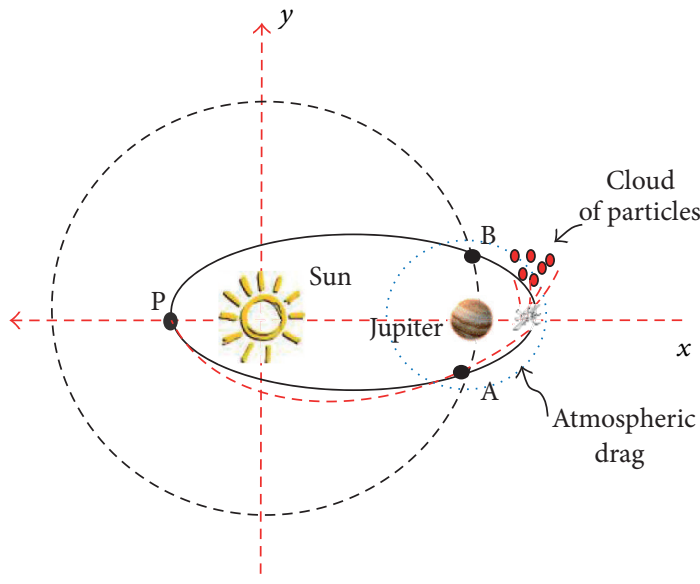

FIGURE 1: Geometry of the maneuver. It is not in scale to visualize better all the steps.

$\rho_{0}$ is the density of the atmosphere at an altitude $h_{0} ; h$ is the altitude of the spacecraft; $H$ is a constant that specifies the decay velocity of the density with the altitude.

The "Jacobian Integral" is a constant of motion that is very important in the restricted three-body problem. It is valid only when the atmospheric drag is not included. In the present paper it is used as a parameter to identify a single trajectory. It is expressed by:

$$
J=E-\omega C=\frac{\dot{X}^{2}+\dot{Y}^{2}}{2}-\frac{X^{2}+Y^{2}}{2}-\frac{1-\mu}{r_{1}}-\frac{\mu}{r_{2}},
$$

where $E$ is the energy; $C$ is the angular momentum; $r_{1}$ is the distance between the spacecraft and the Sun; $r_{2}$ is the distance between the spacecraft and Jupiter; $\omega$ is the angular velocity of the Sun-Jupiter system.

\section{Identifying a Trajectory}

It is very important to define a set of variables that can identify uniquely one Swing-By trajectory. There are several choices that can be used for this task. In the present research the following ones are used [15]:

(a) $\Psi$, the angle of approach. It is the angle formed by the line of the periapsis (line linking the center of Jupiter to the point of the closest approach of the trajectory) and the line connecting the two primaries (Sun-Jupiter). In the rotating system of reference this line is also the horizontal axis;

(b) $J$, the Jacobian constant, expressed by (4). Although this is no longer constant after the inclusion of the atmospheric drag, this parameter is usually used to identify Swing-By trajectories. It is used only to find the velocity of the particle at the periapsis;

(c) $h_{p}$, the altitude of the periapsis for the trajectory around Jupiter.

The choice of these parameters was made based in the literature [15], that used those variables and they showed to be very adequate to describe the problem.
So, the methodology used to compare trajectories considering or not the drag force is based in the steps shown below.

(i) When studying trajectories without the drag force, a numerical integration is made starting at the periapsis and in backward time, until the point $\mathrm{A}$ is reached by the spacecraft (see Figure 1). At this point the spacecraft is assumed to be far enough from Jupiter, so the formulas developed for the two-body celestial mechanics are valid to compute the two-body energy and angular momentum before the close approach for the Sun-spacecraft system. After that, the initial conditions are returned to the periapsis. The cloud of particles is created by making small modifications in the variables $\Psi$ and $h p$. So, the particles are assumed to have values $\Psi-\delta \Psi \leq \Psi \leq \Psi+\delta \Psi$ for the angle of approach and $h_{p}-\delta h_{p} \leq h_{p} \leq h_{p}+\delta h_{p}$ for the altitude of the periapsis. Limits for those increments $\left(\delta \Psi, h_{p}\right)$ are fixed in advance, to define the characteristics of the cloud. Larger values represent a strong explosion that generates a more dispersed cloud. On the other side, smaller values for those two limits represent weaker explosions, with a more concentrated cloud of particles. In this part of the calculations it is assumed that the Jacobian constant is the same for all the particles, at the beginning of the motion. Then numerical integrations are made for each particle, this time in forward time. This integration is performed until the spacecraft collide with the planet or escape from it and reaches a point B (see Figure 1), assumed to be a point that is far enough from Jupiter such that its effects in the motion of the particle are negligible. At this point two-body celestial mechanics are again assumed to be valid to compute the two-body energy and angular momentum after the close approach for the Sun-spacecraft system again;

(ii) The maneuver with the presence of the drag force is similar. The numerical integration starts at periapsis, with the same values for the parameters $J, \Psi$ and $h_{p}$ of the maneuver without drag. The integration of the trajectory of the spacecraft is made again backward in time, now including the drag force, until the spacecraft arrives at the point A. The numerical integration for the particles is performed forward in time, including the drag force, for each particle until they reach the point $B$. The same hypotheses made for the situation without the drag force are made here regarding the initial condition of each particle.

The same quantities are now calculated to measure the effects of the atmosphere: the energy, the angular momentum, the semi-major axis, the eccentricity and the Jacobian constant before and after the Swing-By. This procedure is repeated with and without the presence of the drag force, what makes possible to measure its influence. The results are shown in figures that have the semi-major axis and the eccentricity of the particles of the cloud after the passage by the atmosphere and the corresponding values for the spacecraft before the passage. 


\section{Tisserand's Criterion}

It is known for a long time that, when a particle passes close to a massive celestial body, the orbital elements can be drastically modified. The French astronomer Francois Felix Tisserand established, in 1889, a relationship among the orbital elements that should remain almost unaltered by a close approach. It is an equation, written in the dimensionless canonical system of units, which represents a quantity that should be nearly conserved by the close approach. Thus, two observed bodies are probably the same if they nearly satisfy the Tisserand's Criterion [34]:

$$
\frac{1}{a_{i}}+2 \sqrt{a_{i}\left(1-e_{i}^{2}\right)} \cos i_{i} \approx \frac{1}{a_{0}}+2 \sqrt{a_{0}\left(1-e_{0}^{2}\right)} \cos i_{0},
$$

where $a_{i}, e_{i}$ and $i_{i}$ are the orbital elements of the particle before the maneuver and $a_{0}, e_{0}$ and $i_{0}$ are the orbital elements of the particle after the maneuver. It is important to note that all the orbits involved are planar. It means that there are only two choices for the inclination before and after the close approach: zero or 180 degrees. So, the trigonometric forms presented in this equation $\left(\cos i_{i}\right.$ and $\cos i_{0}$ ) are both minus one or one. In this study, the Tisserand's Criterion will be used to validate the orbits found.

\section{Results}

The results are presented in figures that display the semimajor axis and the eccentricity of the particles of the cloud after the passage for both cases, with and without drag. The legends show the same values for the spacecraft, before the passage. Two values were considered for the Jacobian constant $J:-1.3$ and -1.0 . Those values were chosen based in simulations with different values. Since the Jacobian constant is equivalent to the velocity of the spacecraft at the periapsis, it is important to use values that do not imply in velocities that are not too low, such that too many captures by Jupiter occur; and also those which are not too high, such that the SwingBys have very little effects. Eight values were considered for the angel approach $\Psi: 0^{\circ}, 45^{\circ}, 90^{\circ}, 135^{\circ}, 180^{\circ}, 225^{\circ}, 270^{\circ}, 315^{\circ}$. Those values were chosen in order to cover the whole range of values from 0 to $360^{\circ}$ in equally spaced values, such that there are captures and escapes for the particles, as well as regions with no effects from the Swing-Bys. Figures 2, 3, 4, 5, 6, 7, 8, and 9 show the results, where the semi-major axes are shown in canonical units. Red represents the cloud of particles for the passage where the drag is included and black represents the situation where drag is not included.

There are many conclusions coming from those results. First, it is clear that the inclusion of the atmospheric drag increases the dispersion of the particles of the cloud. It happened for all the situations simulated. Another effect common to all the simulations is the circularization of the orbits. It means that the atmospheric drag reduces the eccentricities of the particles, when compared to the equivalent simulations without the presence of drag. Considering specific cases, it is visible that the maneuvers, in the situation where $\Psi=0^{\circ}$, have no change in the energy of the particle due to the SwingBy. The centers of the black curves, for both values of the
TABLE 1: Amplitude of the dispersions for semi-major axis and eccentricity.

\begin{tabular}{lcccccc}
\hline \multirow{2}{*}{ Figure } & \multirow{2}{*}{$\Psi(\mathrm{deg})$} & $J$ & \multicolumn{2}{c}{ No drag } & \multicolumn{2}{c}{ With drag } \\
& & & $\Delta a$ & $\Delta e$ & $\Delta a$ & $\Delta e$ \\
\hline \multirow{2}{*}{ Figure 2 } & \multirow{2}{*}{0} & -1.3 & 0.27398 & 0.01378 & 0.40155 & 0.04336 \\
& & -1.0 & 0.04844 & 0.03052 & 0.14377 & 0.05914 \\
Figure 3 & \multirow{2}{*}{45} & -1.3 & 0.01231 & 0.00558 & 0.01820 & 0.01735 \\
& & -1.0 & 0.06168 & 0.00105 & 0.08655 & 0.00727 \\
Figure 4 & \multirow{2}{*}{90} & -1.3 & 0.00164 & 0.00180 & 0.00502 & 0.01627 \\
& & -1.0 & 0.06168 & 0.00105 & 0.08655 & 0.00727 \\
Figure 5 & \multirow{2}{*}{135} & -1.3 & 0.00380 & 0.00381 & 0.00952 & 0.02189 \\
& & -1.0 & 0.00357 & 0.00074 & 0.00428 & 0.00338 \\
Figure 6 & \multirow{2}{*}{180} & -1.3 & 0.03220 & 0.00117 & 0.03701 & 0.01684 \\
& & -1.0 & 0.04784 & 0.00245 & 0.08567 & 0.00675 \\
Figure 7 & \multirow{2}{*}{225} & -1.3 & 15.06492 & 0.01648 & 28.89744 & 0.04306 \\
& & -1.0 & 0.06267 & 0.02993 & 0.12365 & 0.04258 \\
Figure 8 & \multirow{2}{*}{270} & -1.3 & 0.01418 & 0.00616 & 0.26598 & 0.05998 \\
& & -1.0 & 0.00419 & 0.01809 & 0.01787 & 0.05396 \\
Figure 9 & \multirow{2}{*}{315} & -1.3 & 0.04569 & 0.01179 & 0.64335 & 0.06859 \\
& & -1.0 & 0.00359 & 0.01621 & 0.01966 & 0.06234 \\
\hline
\end{tabular}

Jacobian constant, have the same keplerian elements of the spacecraft before the passage. The particles are dispersed by the variations of the initial conditions only (angle of approach and periapsis altitude). It is also visible that all the orbits involved are hyperbolic for the situation where $J=-1.0$, since the eccentricities are larger than one and the semi-major axis are positive. For the situations where $J=-1.3$ all the orbits involved are elliptic. These differences come from the higher magnitude of the velocity at the periapsis caused by the increase of the Jacobian constant. The addition of the atmospheric drag has the effect of making an extra loss in the energy, as expected, showed by the decreases of the semimajor axis. It is also visible that, with the increase of the Jacobian constant (and so the velocity at the periapsis) the modifications in the orbit of the spacecraft decreases. This is explained by the fact that the particles and the spacecraft remains a longer time inside the atmosphere in the situations where the velocity is smaller.

For the situations where $=45^{\circ}$, the Swing-By maneuver reduces the energy of the particle. All the orbits before the close approach are hyperbolic and after the close approach they are elliptic, for both values of the Jacobian constant. It means that captures occur in all situations and the particles become part of the Solar System after the close approaches. Once again, the effects are smaller when the velocity increases, for the same reasons already explained. Both values of the semi-major axis and eccentricity are larger for higher velocities at the periapsis.

The cases where $\Psi=90^{\circ}$ represent the maneuvers that give the maximum loss of energy for the particles due to the Swing-by. Once again there are capture trajectories in all the situations, since the particle is in a hyperbolic orbit before the passage and in elliptical orbits after that. Note that for the case $J=-1.0$ the captures resulted in orbits with eccentricity 


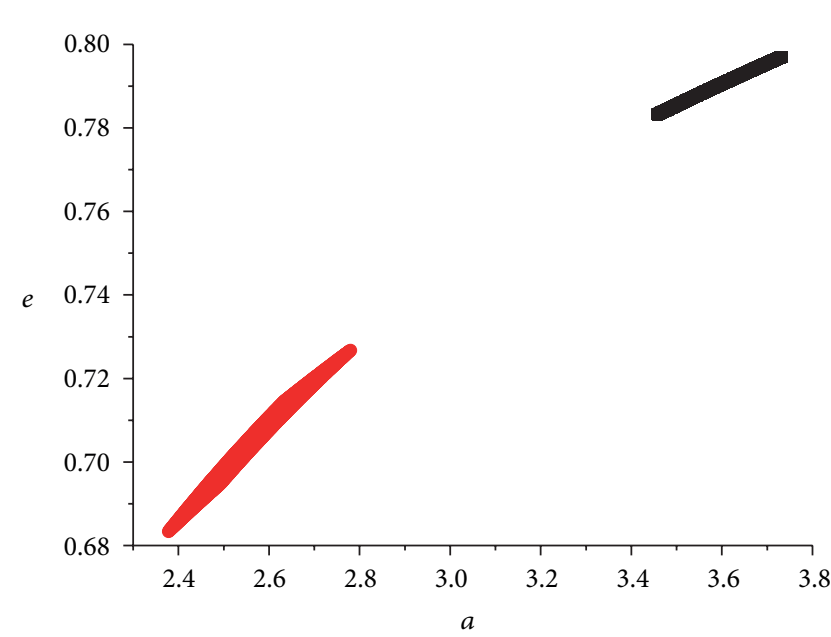

- No drag

- With drag

(a) $J=-1.3$, keplerian elements of the spacecraft before the Swing-By: $a=3.58547, e=0.7898784$

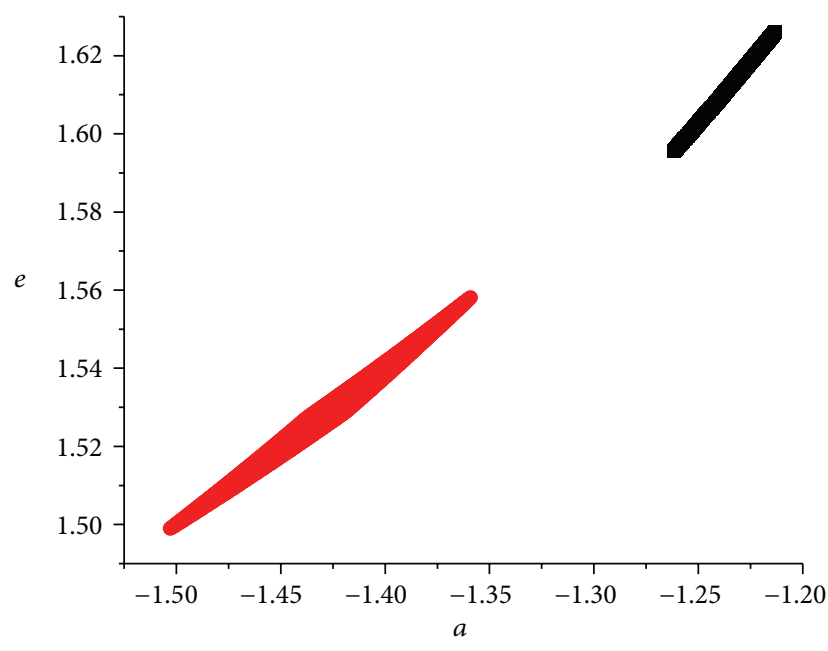

- No drag

- With drag

(b) $J=-1.0$, keplerian elements of the spacecraft before the Swing-By: $a=-1.23775, e=1.610314$

Figure 2: Semi major (a) axis and eccentricity (e) for the particles after the close approach considering $\Psi=0^{\circ}$.

TABLE 2: Orbital parameters and Tisserand's values for a cloud of particles before and after the explosion.

\begin{tabular}{|c|c|c|c|c|c|c|c|}
\hline \multirow{4}{*}{$J$} & \multirow{2}{*}{\multicolumn{3}{|c|}{ Initial orbit (canonical units) }} & \multicolumn{4}{|c|}{ After maneuver (cloud of particles) } \\
\hline & & & & & Tisse & riterion & \\
\hline & \multirow[t]{2}{*}{$\Psi(\mathrm{deg})$} & \multirow[t]{2}{*}{$a$} & \multirow[t]{2}{*}{$e$} & \multirow{2}{*}{$\begin{array}{c}\text { No drag } \\
T_{-}\end{array}$} & \multicolumn{3}{|c|}{ With atmospheric drag } \\
\hline & & & & & $T_{m}$ & $T_{\min }$ & $T_{\max }$ \\
\hline \multirow{8}{*}{-1.0} & 0 & $-1,23775$ & 1,610314 & 2,000569 & 2,06071106 & 2,05025320 & 2,07252358 \\
\hline & 45 & $-0,53638$ & 2,821376 & 2,000065 & 2,06142012 & 2,05096604 & 2,07322829 \\
\hline & 90 & $-0,54698$ & 2,774633 & 2,000082 & 2,06192721 & 2,05148132 & 2,07372668 \\
\hline & 135 & $-1,39075$ & 1,526299 & 2,000611 & 2,06193529 & 2,05149121 & 2,07373280 \\
\hline & 180 & 1,229213 & 0,844421 & 2,001342 & 2,06136489 & 2,05091418 & 2,07316926 \\
\hline & 225 & 0,534676 & 0,995945 & 2,001846 & 2,06073155 & 2,05027747 & 2,07253978 \\
\hline & 270 & 0,545158 & 0,993546 & 2,001829 & 2,06022456 & 2,04977090 & 2,07203293 \\
\hline & 315 & 1,379367 & 0,839495 & 2,001300 & 2,06021643 & 2,04976138 & 2,07202647 \\
\hline \multirow{8}{*}{-1.3} & 0 & 3,58547 & 0,7898784 & 2,601373 & 2,66063381 & 2,65033163 & 2,67227080 \\
\hline & 45 & $-2,03535$ & 1,4746355 & 2,601006 & 2,66105799 & 2,65076288 & 2,67268695 \\
\hline & 90 & $-1,60122$ & 1,6199792 & 2,600942 & 2,66130887 & 2,65102208 & 2,67292885 \\
\hline & 135 & $-26,9689$ & 1,0317580 & 2,601223 & 2,66124006 & 2,65095278 & 2,67286048 \\
\hline & 180 & 0,9678 & 0,6037537 & 2,601733 & 2,66084666 & 2,65055228 & 2,67247478 \\
\hline & 225 & 0,58864 & 0,8084081 & 2,602051 & 2,66047080 & 2,65017124 & 2,67210496 \\
\hline & 270 & 0,54589 & 0,8534019 & 2,602114 & 2,66022005 & 2,64991829 & 2,67185720 \\
\hline & 315 & 0,80375 & 0,6532013 & 2,601834 & 2,66028895 & 2,64998489 & 2,67192855 \\
\hline
\end{tabular}

just below 1.0, so they are almost parabolic orbits and the captures almost did not happen. The cases with $J=-1.3$ have lower velocities, so the captures are stronger and the eccentricities after the close approaches are in the range 0.81 to 0.83 , so not so close to parabolic orbits. It is also noted that the semi-major axis for the particles are larger when the atmospheric drag is included, what is not expected since the atmosphere takes energy from the particles. This situation happened because the maneuvers with $\Psi=90^{\circ}$ cause the largest loss of energy due to the passage by Jupiter, so, if the atmosphere changes this geometry and the trajectories have angles of approach different from that extreme case, there is a reduction in the variation of energy due to the Swing-By. It means that the magnitudes of the energy removed by the atmosphere were smaller than the magnitude of the decreases in the variation of energy due to the fact that the Swing-By occurred out of the extreme geometry.

The situations that have $\Psi=135^{\circ}$ also represent SwingBys that reduces the energy of the particles. For both values of the Jacobian constant all the trajectories ends in captures. 


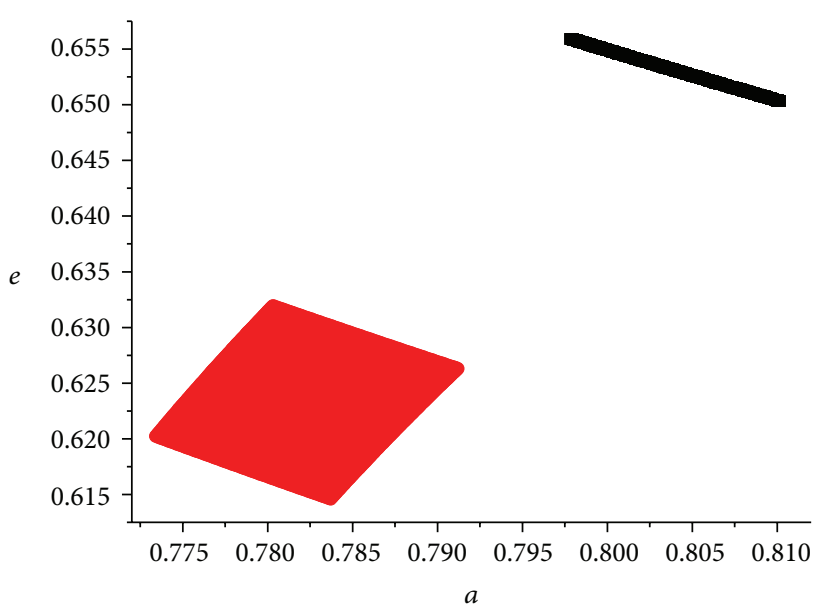

- No drag

- With drag

(a) $J=-1.3$, keplerian elements of the spacecraft before the Swing-By: $a=-2.03535, e=1.4746355$

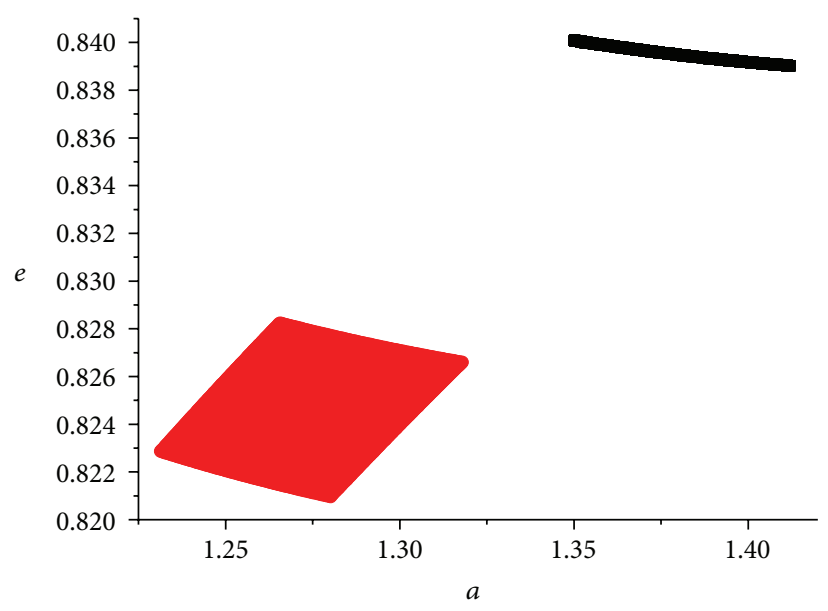

- No drag

- With drag

(b) $J=-1.0$, keplerian elements of the spacecraft before the Swing-By: $a=-0.53638, e=2.821376$

FIGURE 3: Semi major (a) axis and eccentricity (e) for the particles after the close approach considering $\Psi=45^{\circ}$.

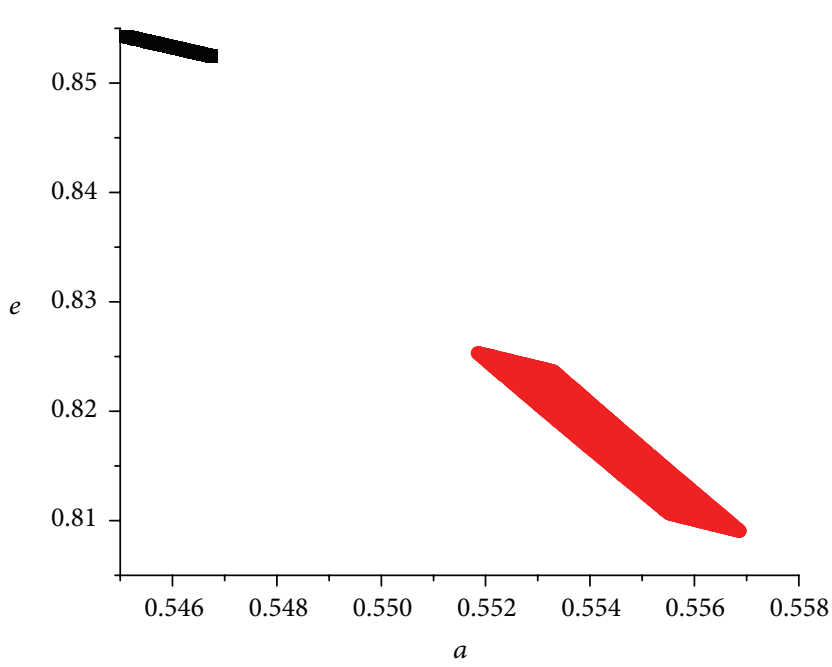

- No drag

- With drag

(a) $J=-1.3$, keplerian elements of the spacecraft before the Swing-By: $a=-1.60122, e=1.6199792$

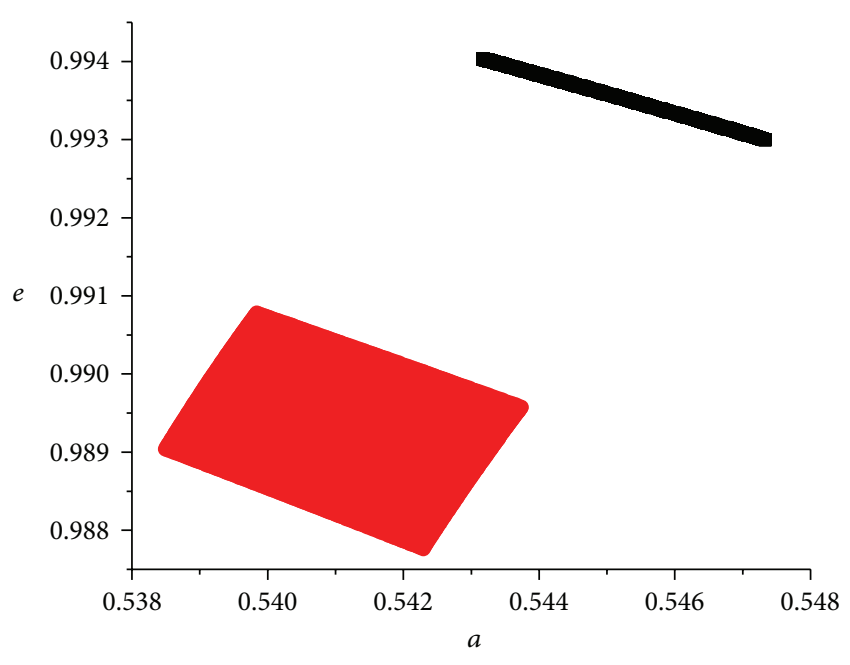

- No drag

- With drag

(b) $J=-1.0$, keplerian elements of the spacecraft before the Swing-By: $a=$ $-0.54698, e=2.774633$

FIGURE 4: Semi major (a) axis and eccentricity (e) for the particles after the close approach considering $\Psi=90^{\circ}$.

Once again the characteristic of having smaller loss of energy when the atmosphere is included occurred, this time for both values of the Jacobian constant. In the case where $J=-1.0$ the captures resulted in orbits with very high eccentricities, near the parabolic orbits again. The cases where $J=-1.3$ have now the characteristic of having almost parabolic orbits before the close approach.

The cases where $\Psi=180^{\circ}$ represent the other geometry where the Swing-By itself does not modify the orbit of the particle, similar to the case $\Psi=0^{\circ}$. All the orbits are elliptic, before and after the close approaches, for both values of the Jacobian constant. Another characteristic of these orbits is that there is an inversion in the change of energy due to the inclusion of the drag force. There are decreases for $J=-1.3$ and increases for $J=-1.0$. This fact occurs due to the sensibility of the orbits in the border line between gains and losses in energy, in terms of the angle of approach. The atmosphere can make small deviations in the orbits and put them in trajectories with angle of approaches a little below $180^{\circ}$, so loosing energy, or a little above $180^{\circ}$, gaining 


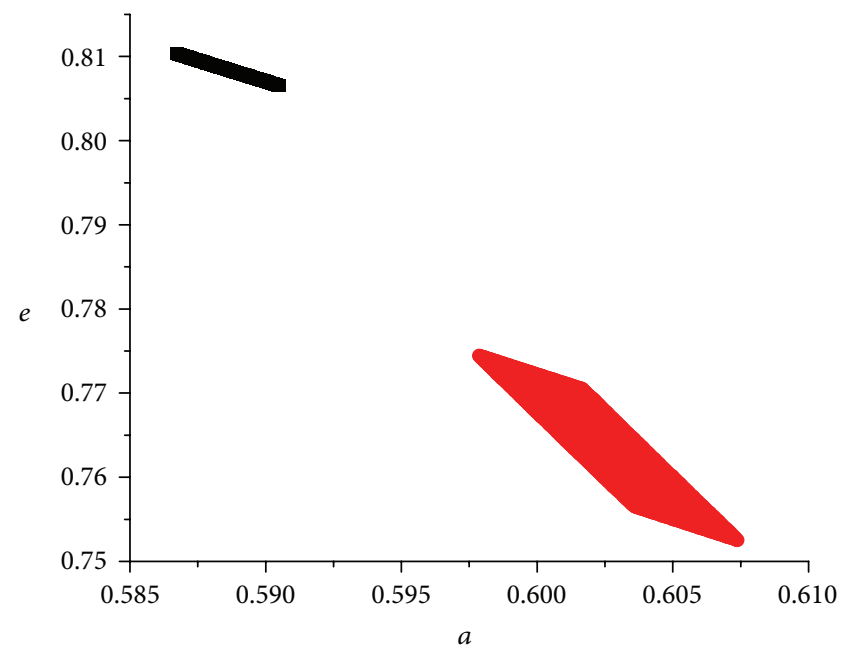

- No drag

- With drag

(a) $J=-1.3$, keplerian elements of the spacecraft before the Swing-By: $a=-26.9689, e=1.0317580$

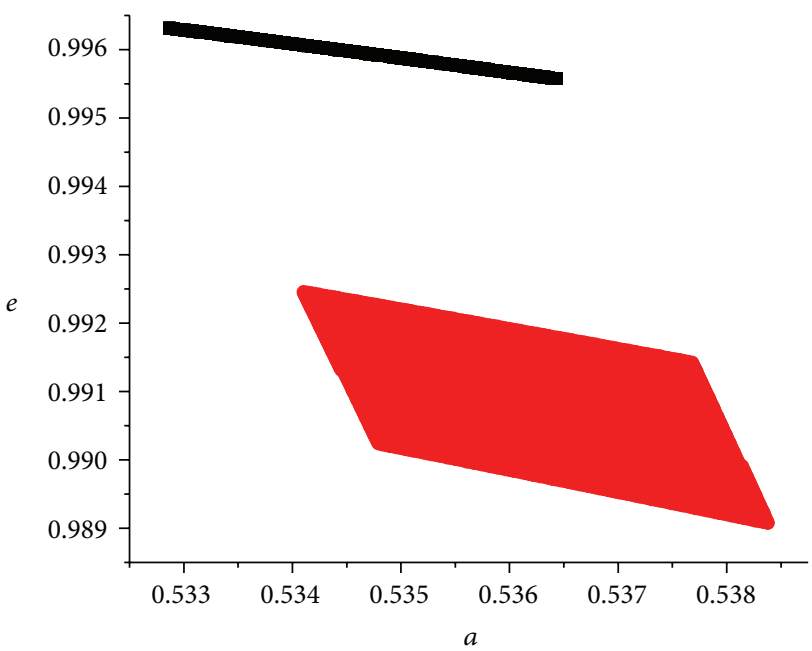

- No drag

- With drag

(b) $J=-1.0$, keplerian elements of the spacecraft before the Swing-By: $a=-1.39075, e=1.526299$

FIGURE 5: Semi major (a) axis and eccentricity (e) for the particles after the close approach considering $\Psi=135^{\circ}$.

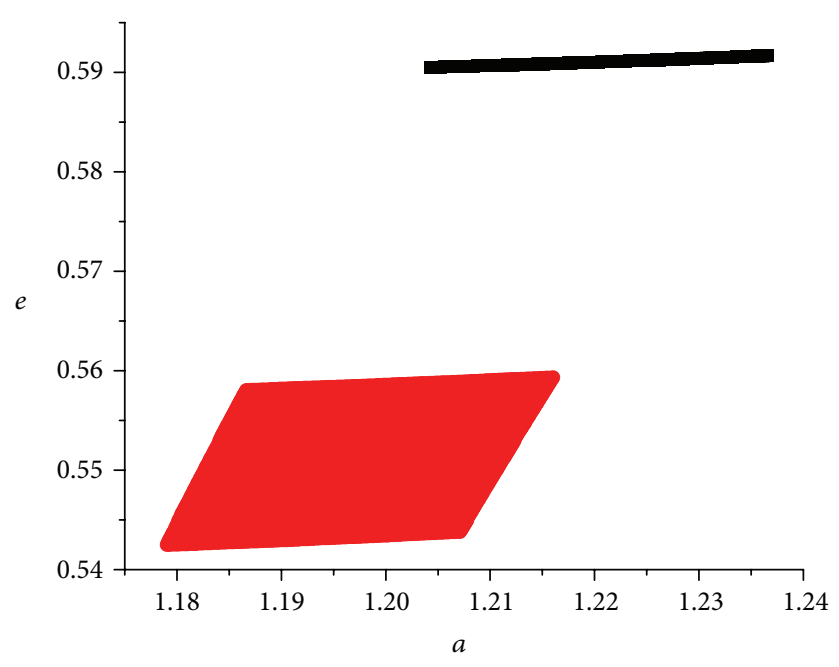

- No drag

- With drag

(a) $J=-1.3$, keplerian elements of the spacecraft before the Swing-By: $a=0.9678, e=0.6037537$

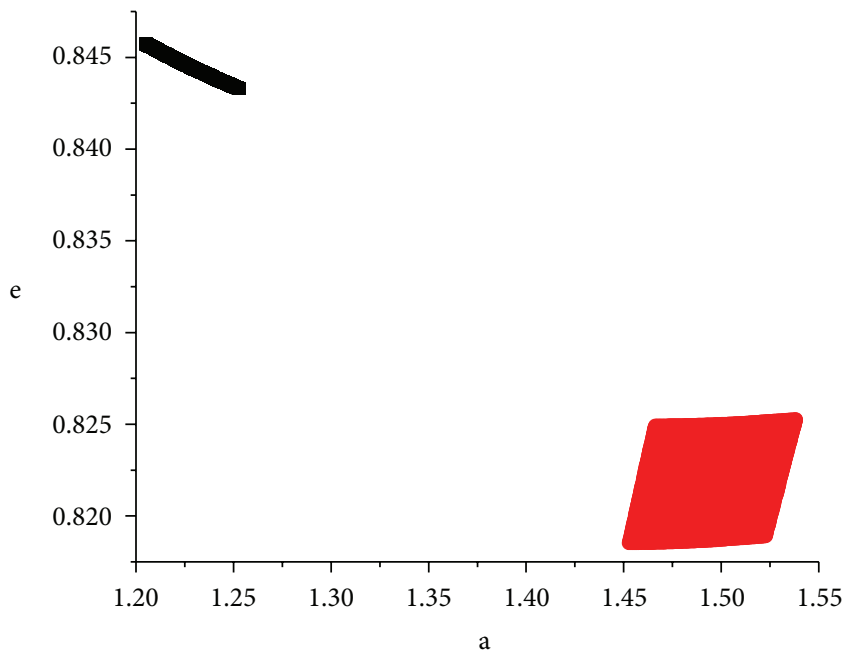

- No drag

- With drag

(b) $J=-1.0$, keplerian elements of the spacecraft before the Swing-By: $a=$ $1.229213, e=0.844421$

FIgURE 6: Semi major (a) axis and eccentricity (e) for the particles after the close approach considering $\Psi=180^{\circ}$.

energy. These differences in the energy variations can be larger than the removal of energy by the atmosphere, causing the unexpected results.

The situations where $\Psi=225^{\circ}$ have also some interesting and peculiar facts. First of all, it is a geometry that increases the energy of the particles. For values of the Jacobian constant equal to -1.0 , there are escape trajectories in all the cases, with the particles leaving closed trajectories around the Sun to go to open trajectories, leaving the Solar System. The escapes occur in the limit, because the orbit before the close approach is almost parabolic, with eccentricity equal to 0.995945 . The cases with $J=-1.3$ are more interesting. Passages not including the drag force also result in escape trajectories, with orbits having eccentricities just above 1.0, so near parabolic orbits. The inclusion of the drag force increases the loss of energy, and these amounts of the increases are large enough to 


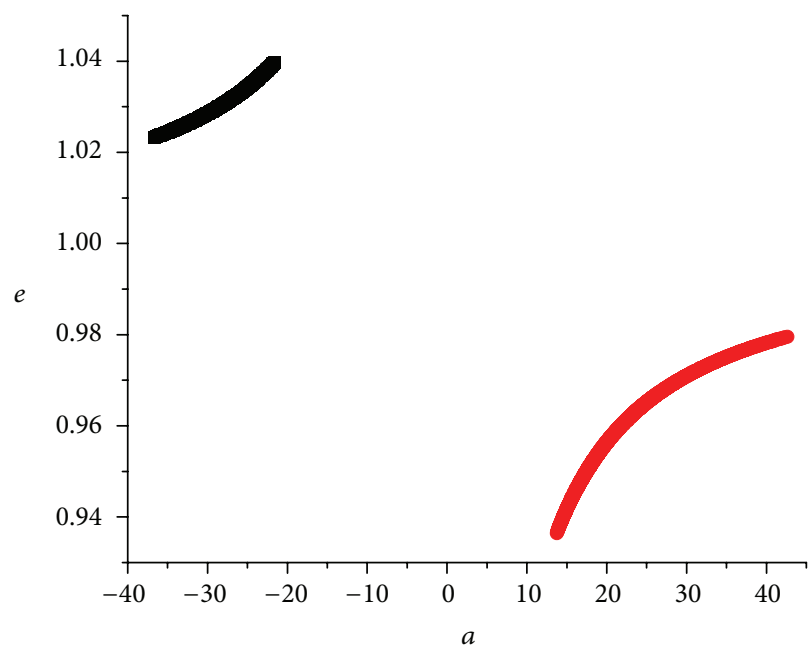

- No drag

- With drag

(a) $J=-1.3$, keplerian elements of the spacecraft before the Swing-By: $a=0.58864, e=0.8084081$

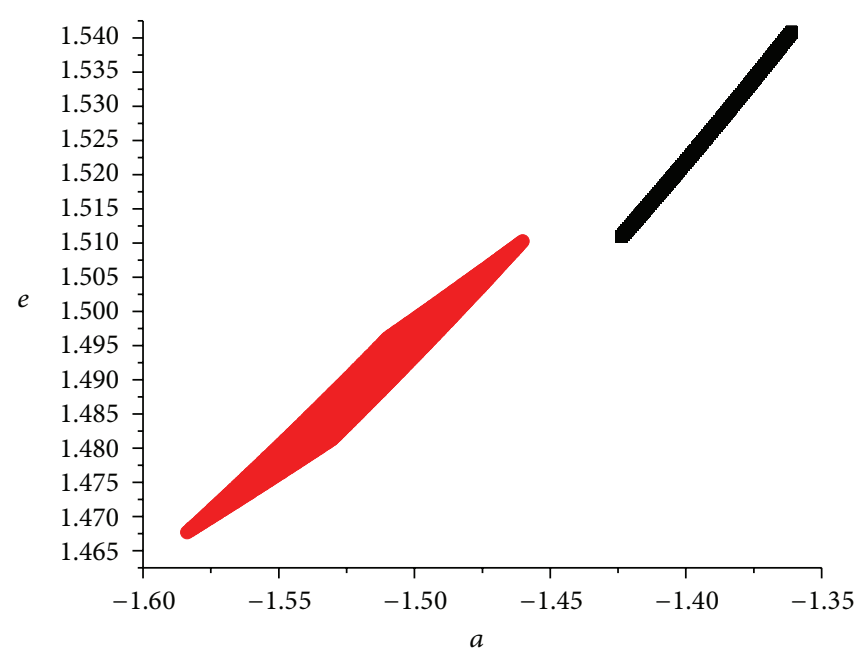

- No drag

- With drag

(b) $J=-1.0$, keplerian elements of the spacecraft before the Swing-By: $a=$ $0.534676, e=0.995945$

FIgURE 7: Semi major (a) axis and eccentricity (e) for the particles after the close approach considering $\Psi=225^{\circ}$.

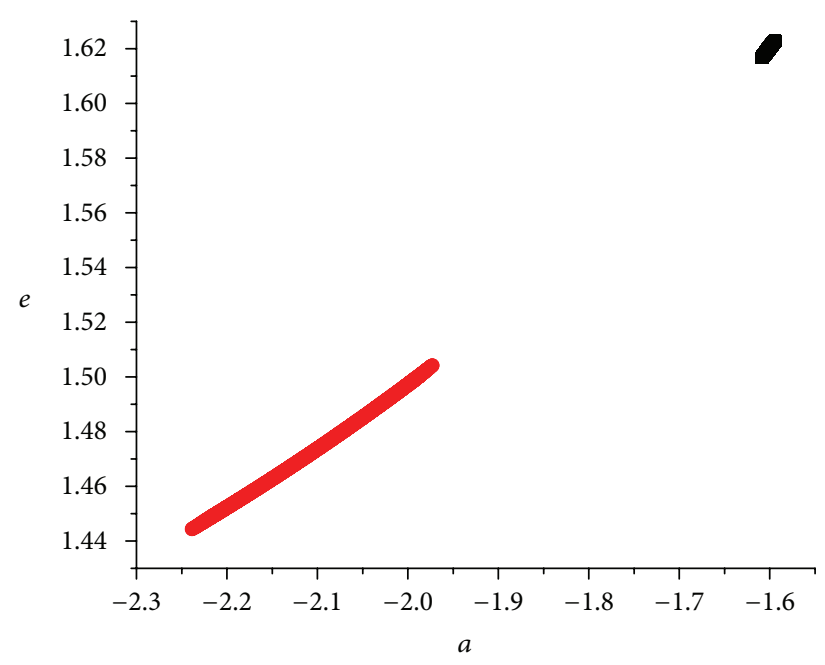

- No drag

- With drag

(a) $J=-1.3$, keplerian elements of the spacecraft before the Swing-By: $a=0.54589, e=0.8534019$

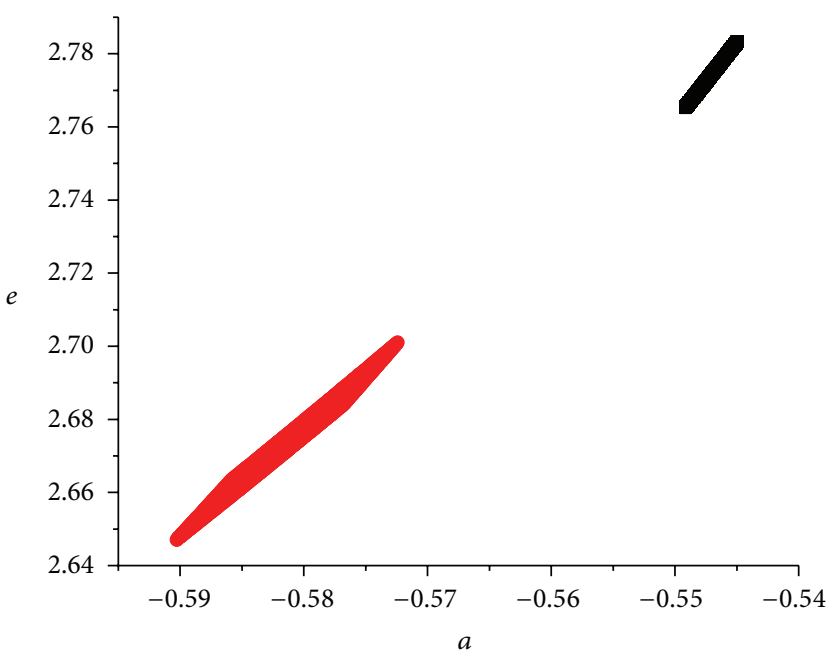

- No drag

- With drag $a=0.545158, e=0.993546$

FIgURE 8: Semi major (a) axis and eccentricity (e) for the particles after the close approach considering $\Psi=270^{\circ}$.

avoid the escapes, so keeping the particles as part of the Solar System. It means that the atmosphere plays an important role in these maneuvers.

The cases with $\Psi=270^{\circ}$ consist in maneuvers that gives the maximum gains in energy for the particles. The result of the larger increases in energy is that now there are only escape trajectories, for both values of the Jacobian constant. It is also noted that the escapes generated trajectories with larger eccentricities, no longer near parabolic orbits. The situation is similar for the last case studied, with $\Psi=315^{\circ}$. There are increases in the energy of the particles in all the cases and all the orbits are hyperbolic.

Table 1 shows the values of the amplitude of the dispersions for both semi-major axis and eccentricity in all cases simulated. It is visible that the inclusion of the atmospheric drag increased the dispersions in all simulations. The increasing factor depends on the geometry of the passage and on the value of the Jacobian constant. 


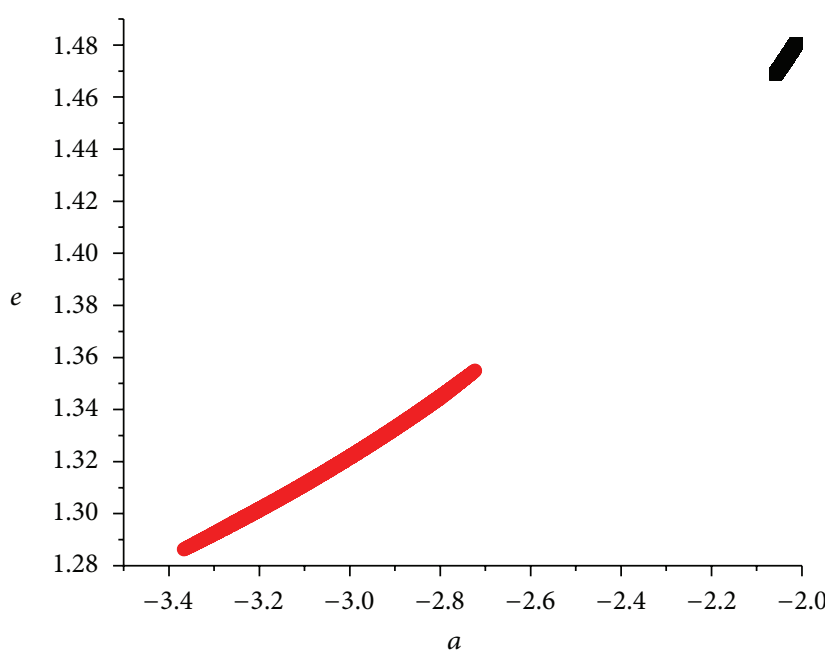

- No drag

- With drag

(a) $J=-1.3$, keplerian elements of the spacecraft before the Swing-By: $a=0.80375, e=0.6532013$

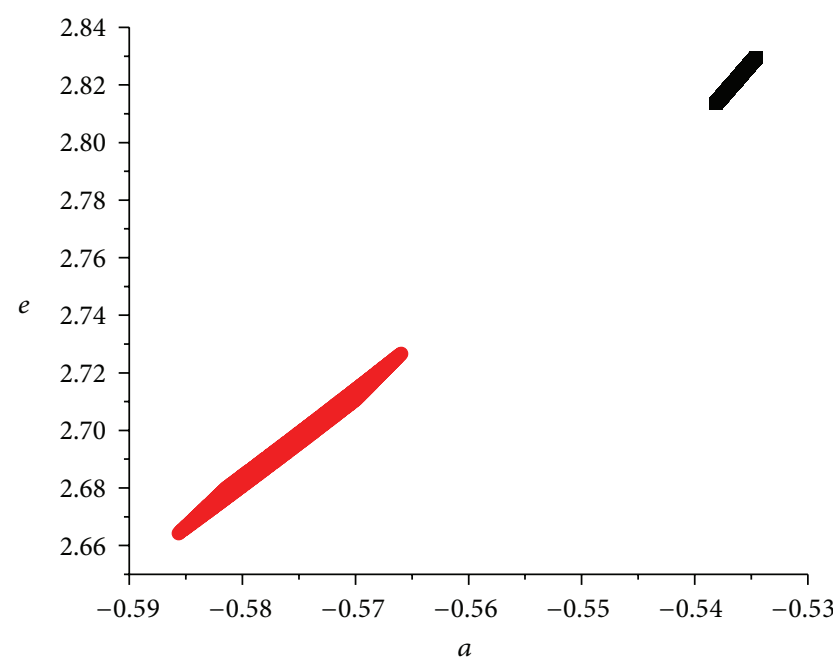

- No drag

- With drag

FIgURE 9: Semi major (a) axis and eccentricity (e) for the particles after the close approach considering $\Psi=315^{\circ}$.

After that, the Tisserand's parameters are evaluated, to verify the orbits found. Table 2 shows the orbital parameters and the Tisserand's values for the spacecraft before the explosion and for the particles of the cloud after the explosion. The values shown are: $J$ (Jacobian constant), $\Psi$ (angle of approach); $a$ (semi-major axis); $e$ (eccentricity); $T_{\text {- }}$ (Tisserand's parameter of the spacecraft before the close approach); $T_{m}$ (the average value of the Tisserand's parameter for the cloud of particles after the explosion); $T_{\min }$ (the minimum value of the Tisserand's parameter for the cloud of particle after the explosion) and $T_{\max }$ (the maximum value for the Tisserand's parameter for the cloud of particles after the explosion). It is possible to observe that the Tisserand's parameters $\left(T_{-}, T_{m}, T_{\min }, T_{\max }\right)$ show slight variations after the second decimal digit due to the atmospheric drag force, so these results are showing the effects of the drag force. In average, the inclusion of the drag force increased the Tisserand's parameter by the value 0.06 . The amplitude of the dispersion is 0.02 .

\section{Conclusions}

A numerical algorithm to measure the effects of the atmosphere in a Swing-By between a planet and a spacecraft that explode during the passage is developed. This algorithm allows the quantification of the results obtained by the inclusion of the drag force and also gives conditions to make predictions in other situations. This technique is then applied to a spacecraft passing by the planet Jupiter. The results give an idea of the behavior of the particles, showing regions where captures and escapes occur, and also quantifying the effects of the atmospheric drag. In general, the presence of the atmosphere causes a reduction in the eccentricities of the orbits of the particles after the Swing-By, so generating orbits that are closer to circular when compared to the situations where drag is not present. This reduction is also quantified by the present algorithm. The semi-major axis of the trajectories are usually reduced by the presence of the atmosphere, but some exceptions exist near the point of maximum loss in energy (angle of approach $90^{\circ}$ ). This fact happens because the losses in energy that come from the deviations from this optimal point for the Swing-By is larger than the energy removed by the atmosphere. Next, the Tisserand's criterion was applied in the maneuvers and the effects of the atmosphere in this parameter were measured, in terms of average and dispersion.

\section{Acknowledgments}

The authors wish to express their appreciation for the support provided by grants 2011/13101-4 and 2012/21023-6, from São Paulo Research Foundation (FAPESP).

\section{References}

[1] M. A. Minovich, "A method for determining interplanetary free-fall reconnaissance trajectories," JPL Technical Memo 312130, JPL, Pasadena, Calif, USA, 1961.

[2] R. L. Dowling, W. J. Kosmann, M. A. Minovitch, and R. W. Ridenoure, "The origin of gravity-propelled interplanetary space travel," in Proceedings of the 41st International Astronautical Federation, GDR. Proceedings, IAA, Dresden, Germany, October 1990.

[3] R. L. Dowling, W. J. Kosmann, M. A. Minovitch, and R. W. Ridenoure, "Gravity propulsion research at UCLA and JPL, 19621964," in Proceedings of the 42st Congress of the International Astronautical Federation, GDR, Dresden, Germany, October 1991. 
[4] G. A. Flandro, "Fast reconnaissance missions to the outer solar system utilizing energy derived from the gravitational field of Jupiter," Astronautica Acta, vol. 12, no. 4, pp. 329-337, 1966.

[5] L. A. D’Amario, D. V. Byrnes, and R. H. Stanford, "Interplanetary trajectory optimization with application to Galileo," Journal of Guidance, Control, and Dynamics, vol. 5, no. 5, pp. 465-471, 1982.

[6] A. A. Sukhanov, "Close approach to sun using gravity assists of the inner planets," Acta Astronautica, vol. 45, no. 4-9, pp. 177$185,1999$.

[7] W. M. Hollister and J. E. Prussing, "Optimum transfer to Mars via Venus," Astronautica Acta, vol. 12, no. 2, pp. 169-179, 1966.

[8] S. A. Striepe and R. D. Braun, "Effects of a venus swingby periapsis burn during an Earth-Mars trajectory," The Journal of the Astronautical Sciences, vol. 39, no. 3, pp. 299-312, 1991.

[9] R. Carvell, "Ulysses-the sun from above and below," Space, vol. 1, pp. 18-55, 1986.

[10] A. F. B. A. Prado, "Orbital control of a satellite using the gravity of the moon," Journal of the Brazilian Society of Mechanical Sciences and Engineering, vol. 28, no. 1, pp. 105-110, 2006.

[11] S. M. Marsh and K. C. Howell, "Double lunar swingby trajectory design," AIAA Paper 88-4289, 1988.

[12] J. K. S. Formiga, "A close approach between a planet and a particle: sun-jupiter system," in Proceedings of the 10th International Conference on System Science and Simulation in Engineering, vol. 1, pp. 32-37, World Scientific and Engineering Academy and Society Press, Penang, Malaysia, 2012.

[13] A. F. B. A. Prado and R. A. Broucke, "Transfer orbits in restricted problem," Journal of Guidance Control and Dynamics, vol. 18, no. 3, pp. 593-598, 1995.

[14] A. F. B. A. Prado and R. A. Broucke, "Transfer orbits in the earth-moon system using a regularized model," Journal of Guidance, Control, and Dynamics, vol. 19, no. 4, pp. 929-933, 1996.

[15] R. A. Broucke, "The celestial mechanics of gravity assist," in Proceedings of the AIAA/AAS Astrodynamics Conference, AIAA paper 88-4220, Minneapolis, Minn, USA, August 1988.

[16] L. A. D’Amario, D. V. Byrnes, L. L. Sacett, and R. H. Stanford, "Optimization of multiple flyby trajectories," in Proceedings of the AAS/AIAA Astrodynamics Specialists Conference, AAS paper 79-162, Provincetown, Mass, USA, June 1979.

[17] L. A. D’Amario, D. V. Byrnes, and R. H. Stanford, "A new method for optimizing multiple-flyby trajectories," Journal of Guidance, Control, and Dynamics, vol. 4, no. 6, pp. 591-596, 1981.

[18] D. Dunham and S. Davis, "Optimization of a multiple lunarswing-by trajectory sequence," Journal of Astronautical Sciences, vol. 33, no. 3, pp. 275-288, 1985.

[19] N. J. Strange and J. M. Longuski, "Graphical method for gravityassist trajectory design," Journal of Spacecraft and Rockets, vol. 39, no. 1, pp. 9-16, 2002.

[20] T. T. McConaghy, T. J. Debban, A. E. Petropoulos, and J. M. Longuski, "Design and optimization of low-thrust trajectories with gravity assists," Journal of Spacecraft and Rockets, vol. 40, no. 3, pp. 380-387, 2003.

[21] A. F. B. A. Prado, "Powered swing-by", Journal of Guidance, Control, and Dynamics, vol. 19, no. 5, pp. 1142-1147, 1997.

[22] A. F. B. A. Prado, "Close-approach trajectories in the elliptic restricted problem," Journal of Guidance, Control, and Dynamics, vol. 20, no. 4, pp. 797-802, 1997.

[23] A. F. B. A. Prado and R. A. Broucke, "Effects of atmospheric drag in swing-by trajectory," Acta Astronautica, vol. 36, no. 6, pp. 285-290, 1995.
[24] W. Schulz, A. F. B. A. Prado, and R. Vilhena de Moraes, "An analytical and numerical study of plane change maneuvers using aerodynamic force," The Journal of the Astronautical Sciences, vol. 50, no. 3, pp. 289-303, 2002.

[25] V. G. Szebehely, Theory of Orbits, Academic Press, New York, NY, USA, 1967.

[26] V. M. Gomes and A. F. B. A. Prado, "Swing-by maneuvers for a cloud of particles with planets of the solar system," WSEAS Transactions on Applied and Theoretical Mechanics, vol. 3, no. 11, pp. 869-878, 2008.

[27] V. M. Gomes and A. F. B. A. Prado, "A study of the impact of the initial energy in a close approach of a cloud of particles," WSEAS Transactions on Mathematics, vol. 9, no. 10, pp. 811-820, 2010.

[28] A. Carusi and F. Pozzi, "A new method for close encounter computation," The Moon and the Planets, vol. 19, no. 1, pp. 65-70, 1978.

[29] A. Carusi and G. B. Valsecchi, "Effects of a close encounter with Jupiter on different populations of planet-crossing objects," The Moon and the Planets, vol. 22, no. 2, pp. 133-139, 1980.

[30] A. Carusi, G. B. Valsechi, and R. Greenberg, "Planetary close encounters: geometry of approach and post-encounter orbital parameters," Celestial Mechanics and Dynamical Astronomy, vol. 49, no. 2, pp. 111-131, 1990.

[31] J. E. Dale and M. B. Davies, "Collisions and close encounters involving massive main-sequence stars," Monthly Notices of the Royal Astronomical Society, vol. 366, no. 4, pp. 1424-1436, 2006.

[32] L. Dong, A. R. Frederic, and S. L. Shapiro, "Collisions and close encounters between massive main-sequence stars," Astrophysical Journal, vol. 412, no. 2, pp. 593-561, 1993.

[33] E. Everhat, "Close encounters of comets and planets," Astronomical Journal, vol. 74, no. 5, 1969.

[34] R. H. Battin, An Introduction to the Mathematics and Models of Astrodynamics, The American Institute of Aeronautics and Astronautics, New York, NY, USA, 1987. 


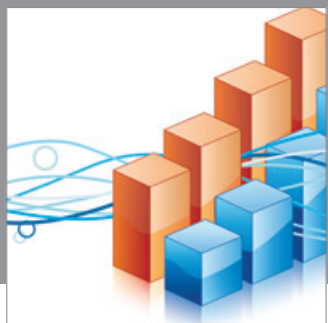

Advances in

Operations Research

mansans

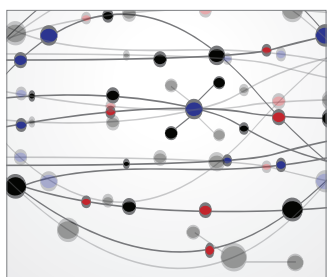

The Scientific World Journal
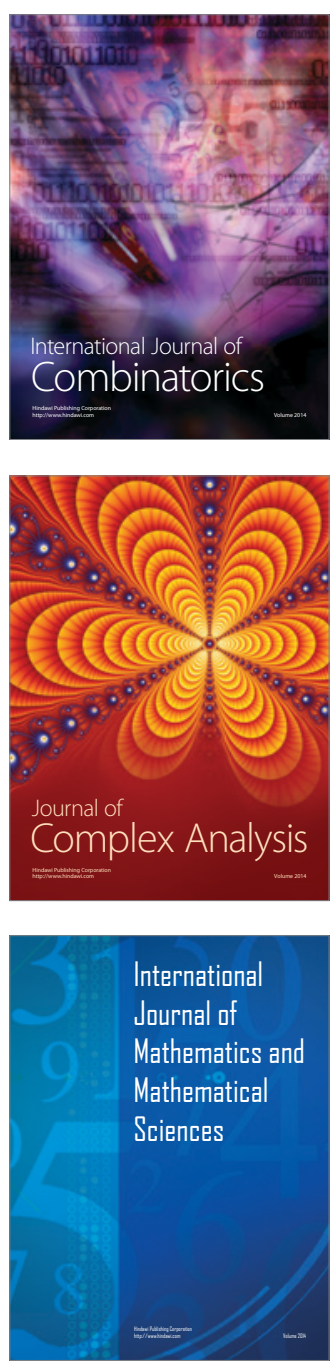
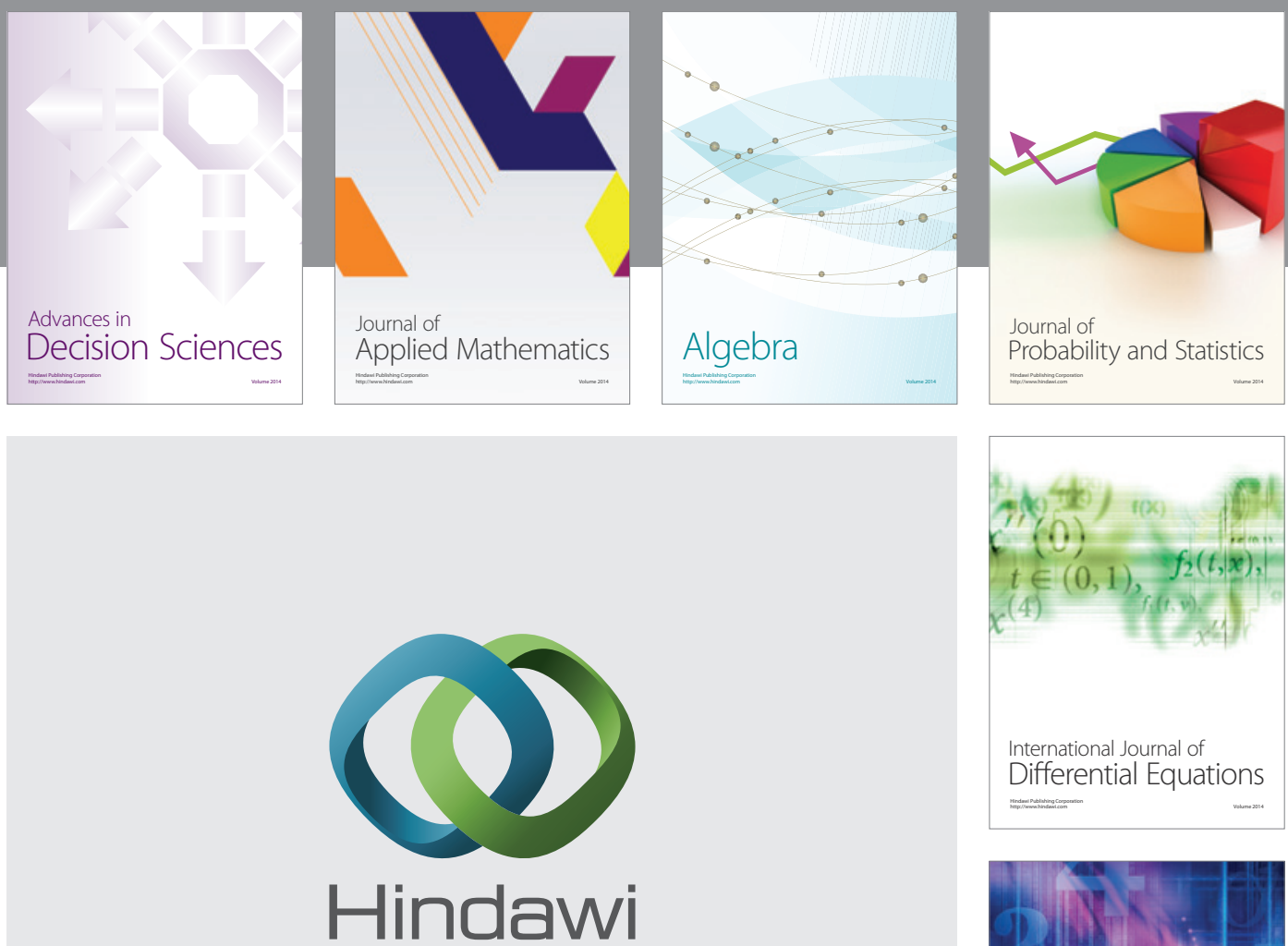

Submit your manuscripts at http://www.hindawi.com
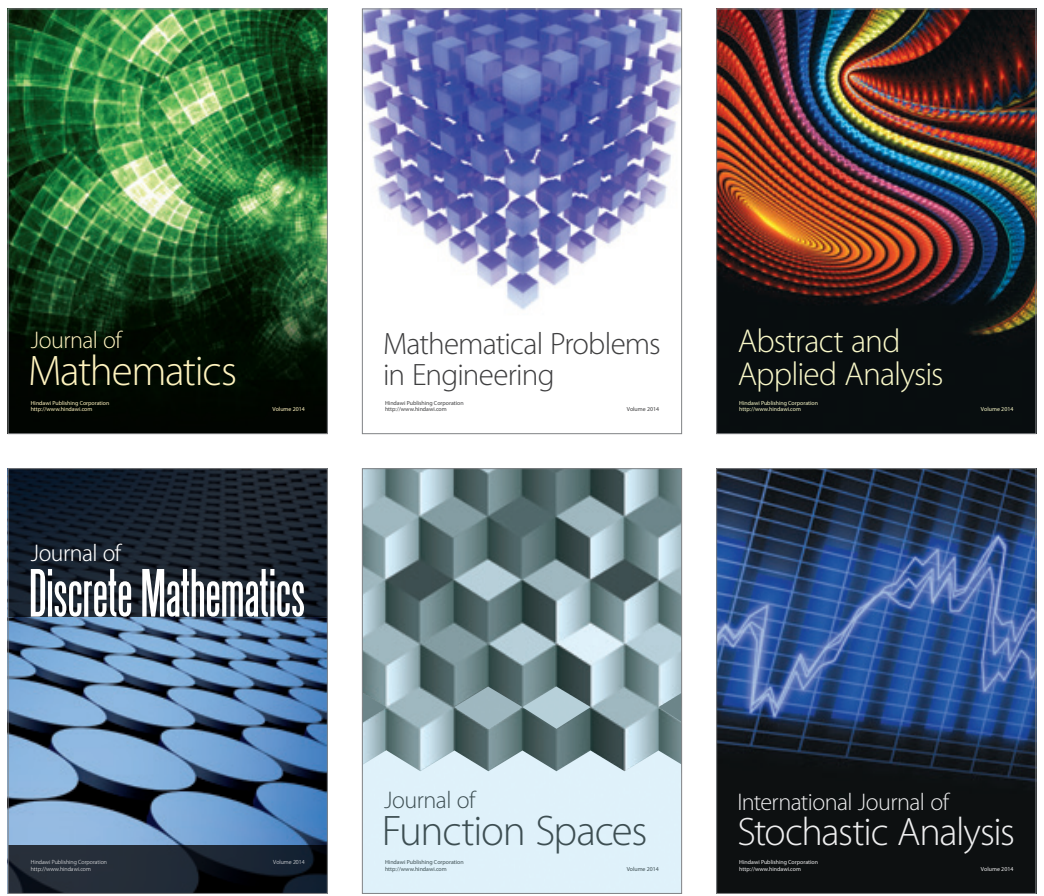

Journal of

Function Spaces

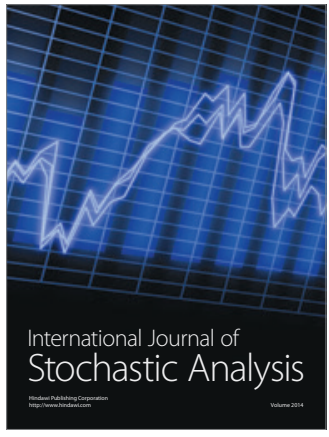

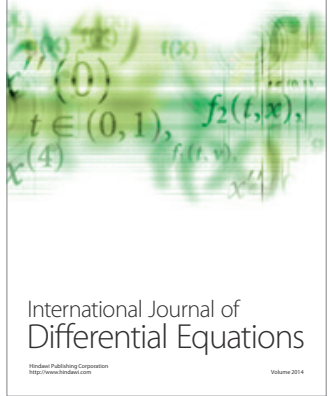
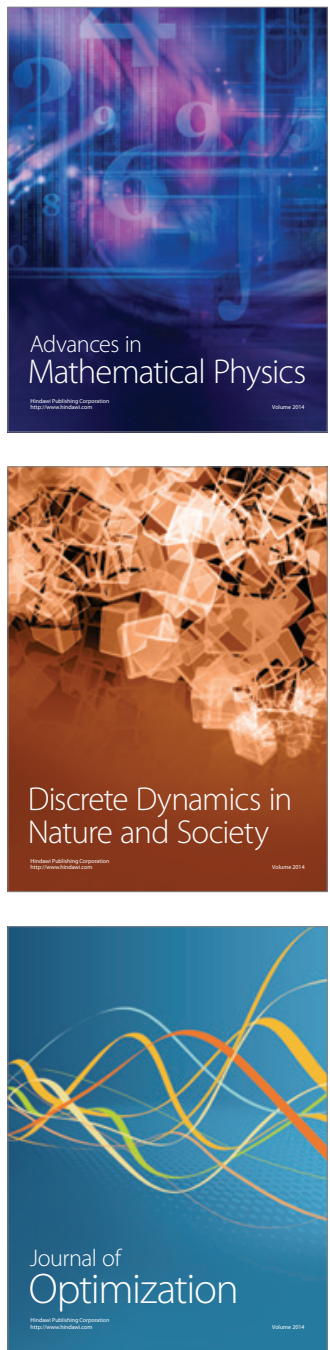